\title{
IMPROVEMENTS IN CVD DIAMOND PROPERTIES FOR RADIOTHERAPY DOSIMETRY
}

\author{
C. De Angelis ${ }^{1, *}$, M. Bucciolini ${ }^{2}$, M. Casati ${ }^{2}$, I. Løvik ${ }^{2}$, M. Bruzzi ${ }^{3}$, S. Lagomarsino ${ }^{3}$, S. Sciortino ${ }^{3}$ and \\ S. Onori ${ }^{1}$ \\ ${ }^{1}$ Istituto Superiore di Sanità and INFN, Viale Regina Elena 299, 00161 Roma, Italy \\ ${ }^{2}$ Dipartimento di Fisiopatologia Clinica dell'Università and INFN, Viale Morgagni 85, \\ 50134 Firenze, Italy \\ ${ }^{3}$ Dipartimento di Energetica dell’Università and INFN, Via Santa Marta 3, 50139 Firenze, Italy
}

\begin{abstract}
The goal of this work was to compare the behaviour of a chemical vapour deposited (CVD) diamond sample, grown at the University of Florence using a local procedure, with that of a commercial CVD diamond. The comparison was performed exposing both systems to $25 \mathrm{MV}$ photons and measuring the current response during irradiation. Properties of dosimetric interest such as stability of response, dose rate dependence and rise time were investigated. After a preliminary study, which evidenced better performances of the commercial device with respect to the local CVD diamond, the latter was irradiated with a high fluence of fast neutrons. As a result of the neutron treatment, the quality of the CVD home-made diamond has been improved to match with that of the commercial dosemeter.
\end{abstract}

\section{INTRODUCTION}

The use of conformal radiotherapy techniques, such as hadron therapy and intensity modulated radiotherapy (IMRT), is imposing increasing demands on dosimetry systems. In particular, high sensitivity, small dimensions, fast rise time, energy and dose rate independence of response and tissue equivalence are the main requirements. Among possible dosimetry devices, diamond synthesised by chemical vapour deposition (CVD) is considered with interest since its characteristics partially satisfy the above reqirements. Indeed, diamond material, because of its strong bonds in the crystal structure, is highly resistant to radiation, the high carrier mobility can result in a fast response, has a good tissue equivalence ( $Z=6$ for diamond and $Z=7.5$ for tissue) as well as a high sensitivity. Moreover, thanks to the significant progress in the synthetic diamond production technology, samples of high quality, in terms of controlled impurity concentration, and of small sizes can be grown. The CVD diamond has been extensively studied as a nuclear radiation detector for applications both in high-energy physics experiments and in the nuclear industry domain. Only recently, some works have also shown the potential suitability of this system for medical dosimetry applications ${ }^{(1-6)}$. Nevertheless, in the authors opinion, efforts have still to be expended in order to improve the properties of CVD diamonds for radiotherapy dosimetry applications, mainly regarding stability of response and rise time.

This work aims to make a contribution to the comprehension of the behaviour of diamond

*Corresponding author: cinzia.deangelis@iss.it detectors when used in medical applications. In particular, it suggests the use of fast-hadrons pre-irradiation $^{(7)}$ as a potential tool to improve dosimetry performances. The study was focused on the behaviour of a CVD device grown at the University of Florence and was performed using a $25 \mathrm{MV}$ photon beam. A preliminary characterisation of the sample was carried out investigating features of dosimetric interest, mainly rise time and stability of response, sensitivity and dose rate dependence. Successively, the sample was irradiated with high fluence neutrons. The results obtained before and after neutron exposure were compared with those from a CVD sample produced by De Beers Industrial Diamond Ltd (UK).

\section{MATERIALS AND METHODS}

Two CVD diamonds were investigated: the first one, labelled F56, was produced at the University of Florence using a proprietary reactor (details about deposition apparatus and process are reported elsewhere $\left.{ }^{(8)}\right)$. The sample had a surface area of $13.4 \mathrm{~mm}^{2}$ and a thickness of $70 \mu \mathrm{m}$. Circular electric contacts with a diameter of $2 \mathrm{~mm}$ were performed by evaporating $\mathrm{Au}$ on the front and back surfaces. The sample showed a leakage current of $0.65 \mathrm{pA}$ at $100 \mathrm{~V}$ bias. The other sample, labelled $\mathrm{D}(\mathrm{Ti}-\mathrm{Au})$, was a commercial De Beers Industrial Diamond Ltd (UK) film, not primed by the manufacturer. Detector volume was $25 \mathrm{~mm}^{2} \times 300 \mu \mathrm{m}$ and it was provided with Ti/ $\mathrm{Au}$ ohmic electric contacts of $2 \times 2.2 \mathrm{~mm}^{2}$, manufactured at Rutgers University, USA. The sample showed a leakage current of $12 \mathrm{pA}$ at $100 \mathrm{~V}$ bias. Both detectors showed a resistivity of $\sim 10^{13}$ $10^{14} \Omega \mathrm{cm}$. 
IMPROVEMENTS IN CVD DIAMOND PROPERTIES

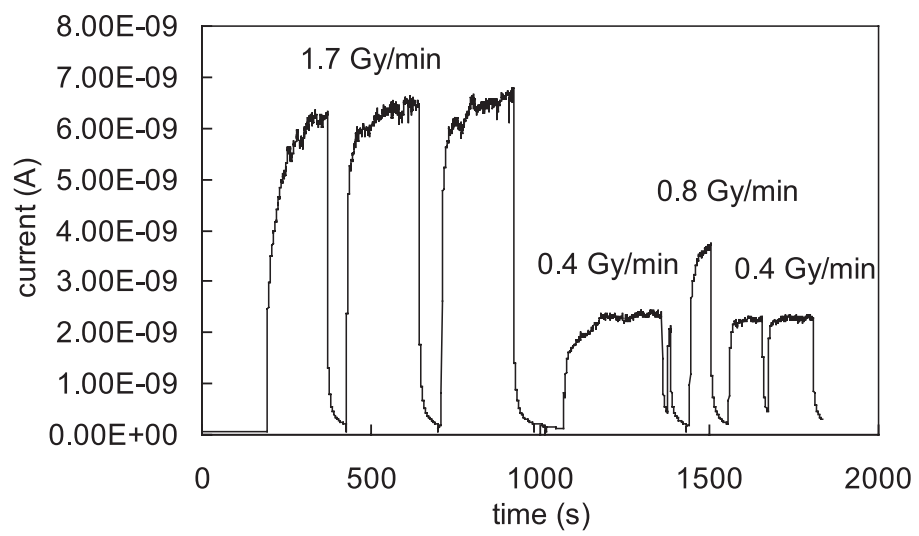

Figure 1. Current response vs. time, at different dose rates, for F56 as grown.

The dosimetric characteristics of the samples were investigated in a $25 \mathrm{MV}$ photon beam produced by a SL25 Elekta LINAC, operating in the Radiotherapy Unit of the Clinical Physiopathology Department of the University of Florence. Dose rates ranging from 1 to $8 \mathrm{~Gy} \mathrm{~min}^{-1}$ were obtained changing both the SSD (source-to-surface distance) from 75 to $140 \mathrm{~cm}$ and the pulse repetition frequency (varying the beam delivery from 100 to $400 \mathrm{MU} \mathrm{min}{ }^{-1}$ ).

For irradiation, the devices were placed into a PMMA phantom at $5 \mathrm{~cm}$ water equivalent depth. To avoid contamination of the detector signal by ambient light, the devices were encapsulated in a black plastic cover. Both dosemeters were connected to a Keithley 6517A electrometer and current signal was measured during exposure. Polarization voltage was set at $100 \mathrm{~V}$.

Irradiation to neutrons was performed at the cyclotron facility of the Institute of Nuclear Research of the Hungarian Academy of Sciences, Debrecen, Hungary. A fluence of $5 \times 10^{14} \mathrm{~cm}^{-2}$ of $1 \mathrm{MeV}$ equivalent neutrons was used.

\section{RESULTS AND DISCUSSION}

Stability of response, dose rate dependence, sensitivity and rise time were investigated. As for stability of response, a quite different behaviour was detected for $\mathrm{F} 56$ and $\mathrm{D}(\mathrm{Ti}-\mathrm{Au})$ samples. In both devices the radiation-induced current increased with cumulated dose but, while for $\mathrm{D}(\mathrm{Ti}-\mathrm{Au})$ a stable response was obtained after a pre-irradiation dose of $\sim 30 \mathrm{~Gy}$ (at a dose rate of $1.7 \mathrm{~Gy} \mathrm{~min}^{-1}$ ), for F56 the current never achieved an equilibrium value even after a cumulated dose of $\sim 120$ Gy (at a dose rate of $1.7 \mathrm{~Gy} \mathrm{~min}^{-1}$ ). In that dose range the increment of the signal was $\sim 0.2 \% \mathrm{~Gy}^{-1}$. Also, fluctuations in the two detector responses were not comparable. Typically, fluctuations were within $0.5 \%$ for $\mathrm{D}(\mathrm{Ti}-\mathrm{Au})$ but one order of magnitude higher for F56, indicating a high instability of the sample. This behaviour suggests a massive presence of shallow centres in the F56 diamond material, produced during the growth process, acting as traps for free electrons and holes and are unstable at room temperature. The detector stability was found to depend on dose rate. The effect is shown in Figure 1 and Figure 2 reporting the variation of the detector current with irradiation time for samples F56 and $\mathrm{D}(\mathrm{Ti}-\mathrm{Au})$, respectively, with the measurements performed at different dose rate values. In particular, for the commercial sample it was found that the equilibrium is lost if dose rate is changed; if it is increased a further pumping stage is required, whereas on lowering the dose rate the sample is over-saturated. For F56, a decrease in the slope of current vs. time curve is observed if the dose rate is decreased. Dose rate dependence of response was studied in the range $0.8-3.5 \mathrm{~Gy} \mathrm{~min}^{-1}$, changing the pulse repetition frequency. For $\mathrm{D}(\mathrm{Ti}-\mathrm{Au})$ the equilibrium current was measured, while for F56 it assumed an equilibrium value when variations in the current were $<2 \%$. For both samples the current, $I$, was found to be quasilinear with dose rate, $D_{\mathrm{r}}$, following the Fowler relation $^{(9)} I \propto D_{\mathrm{r}}^{\Delta}$ with $\Delta_{\mathrm{D}(\mathrm{Ti}-\mathrm{Au})}=0.90 \pm 0.01$ and $\Delta_{\mathrm{F} 56}=0.80 \pm 0.02$. The uncertainty in $\Delta$ values was evaluated as the uncertainty in the slope of the fit. For F56, the uncertainty was higher due to the instability of the detector current.

The sensitivity of the devices was determined at $1.7 \mathrm{~Gy} \mathrm{~min}^{-1}$ dose rate with a $100 \mathrm{~V}$ bias. For $\mathrm{D}(\mathrm{Ti}-\mathrm{Au})$ a sensitivity of $11.7 \mu \mathrm{C} \mathrm{Gy}{ }^{-1} \cdot \mathrm{g}^{-1}$ ) was obtained, after the system had reached a stable current while for F56 a value of $\sim 85.8 \mu \mathrm{C} \mathrm{Gy}^{-1} \cdot \mathrm{g}^{-1}$ was found after an accumulated dose of $120 \mathrm{~Gy}$, when however the current was not yet completely stable.

The signal rise time, evaluated as the time required to reach the $90 \%$ of the equilibrium current, is an 


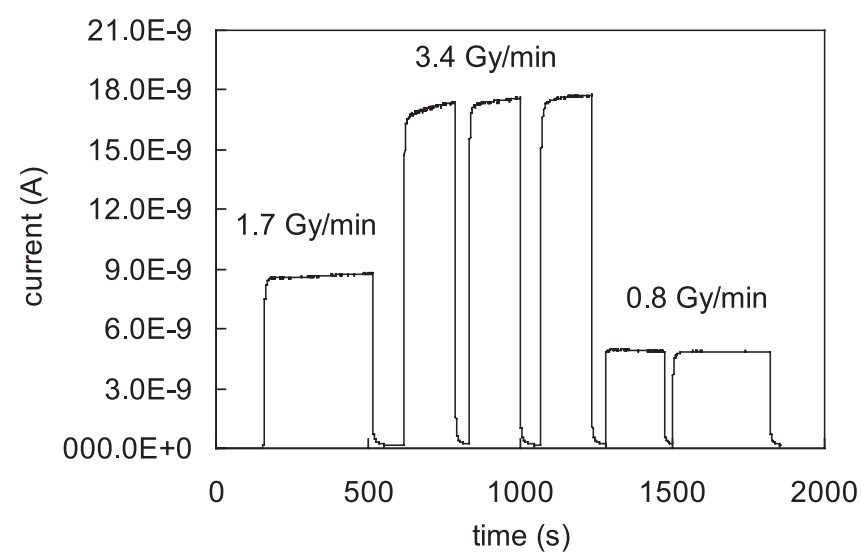

Figure 2. Current response vs. time, at different dose rates, for $\mathrm{D}(\mathrm{Ti}-\mathrm{Au})$ after a cumulated dose of $20 \mathrm{~Gy}$.

important parameter, especially in IMRT where low dose segments from a few monitor units can be employed. A reliable dose measurement requires CVD detectors characterised by a fast response, $<1 \mathrm{~s}$. Because of the lack of a steady signal only an estimate of the rise time for F56 was possible leading to a value of $\sim 20 \mathrm{~s}$, while values ranging from 4.5 to $7 \mathrm{~s}$ were observed for $\mathrm{D}(\mathrm{Ti}-\mathrm{Au})$ at different dose rates in the range $1.5-4 \mathrm{~Gy}^{-1}$.

These results evidenced better performances of the commercial with respect to the local CVD diamond, in particular regarding rise time and stability of response.

To improve the characteristics of the Florence diamond an irradiation with high fluence neutrons was performed. In fact, in a previous work some of the present authors ${ }^{(7,10)}$ already proved an improvement in the dosimetric properties of CVD diamond as a consequence of neutron irradiation. After neutron irradiation, a further investigation was carried out. The resistivity of F56 did not change significantly. As expected, a sensitivity decrease of $\sim 8$ times was observed but also a clear improvement of the performances in terms of stability and rise time of the response was found. A dose of $\sim 50$ Gy was indeed sufficient to obtain stability of the response. Moreover, it was observed that the dark current of the device did not change after neutron irradiation. A qualitative explanation of this effect is that radiation induces recombination centres and deep traps in the material bulk, which partially compensate the native shallower traps responsible for priming. This has a beneficial effect on stability, as native defects which are active close to room temperature and thus contribute to trapping, are deactivated. The slow rise and decay times, which are due to trapping/detrapping of carriers at such shallow levels, thus disappear after neutron irradiation. In Figure 3, the dynamic response of F56 is compared to the behavior of the
$\mathrm{D}(\mathrm{Ti}-\mathrm{Au})$ film. Signal stability and pumping effect are now quite similar for the two detectors. The difference in current signal is due to the higher active volume of the $\mathrm{D}(\mathrm{Ti}-\mathrm{Au})$ sample, the sensitivity of the Florence sample being now quite similar to that of the commercial diamond as well as to the different dose rates used for irradiation. In addition, it should be noted that in successive measurement runs a lower dose of pumping was necessary for F56 to reach the equilibrium current with respect to the first session. This effect, also observed for $\mathrm{D}(\mathrm{Ti}-\mathrm{Au})$, indicates the existence of an amount of deep traps quite stable at room temperature. A similar behaviour was reported by some of the present authors ${ }^{(11)}$ with PTW natural diamond detectors for which a daily pre-irradiation dose lower than that initially required to get stability is necessary if irradiation runs are performed on successive days.

Also, the dose rate dependence of F56 improved after neutron irradiation. A $\Delta$ value of $0.89 \pm 0.01$ was obtained, close to the 0.90 value for the commercial sample.

Figure 4 reports the values of the rise time obtained at different cumulated doses both for F56, after neutron irradiation, and $\mathrm{D}(\mathrm{Ti}-\mathrm{Au})$. Data were derived from different irradiation runs performed at different dose rate values. In particular, for F56 the data obtained in two runs performed on two successive days are shown. To stabilize the response, the detector was pre-irradiated at 50 Gy on the first day of measurement and at $20 \mathrm{~Gy}$ on the second day. In a conservative approach, the uncertainty in the rise time was stated to be equal to the sampling time $(0.1 \mathrm{~s})$. From Figure 4, two effects are clearly evident. The first is the tendency to a reduction of the rise time with the cumulated dose. The second is that a fast response was observed for F56 as well. For both detectors there was a large scatter of the data that could be due to the different dose rates employed in 
IMPROVEMENTS IN CVD DIAMOND PROPERTIES

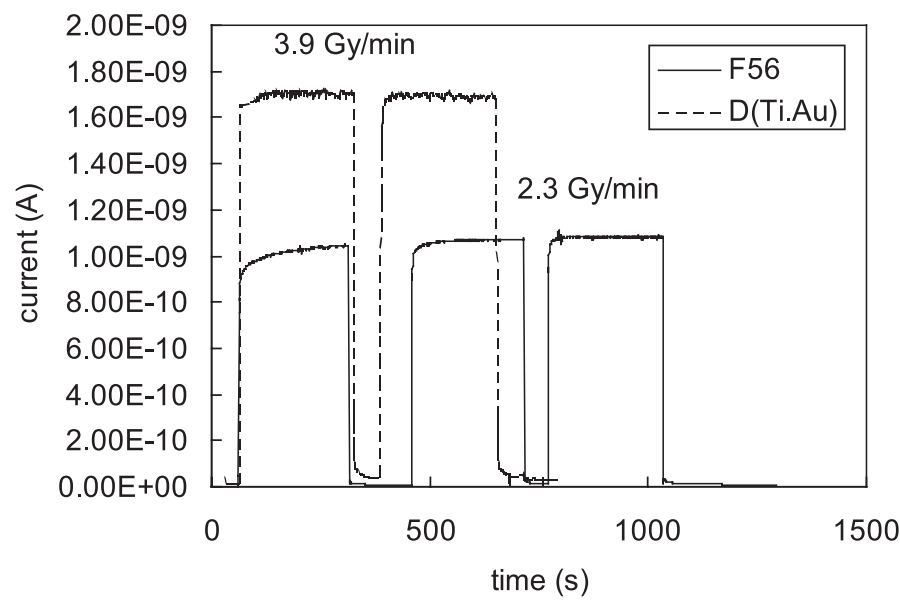

Figure 3. Current response vs. time, at different dose rates, for F56, after the neutron irradiation and D(Ti-Au) detectors. The $\mathrm{D}(\mathrm{Ti}-\mathrm{Au})$ signal is divided by 15 .

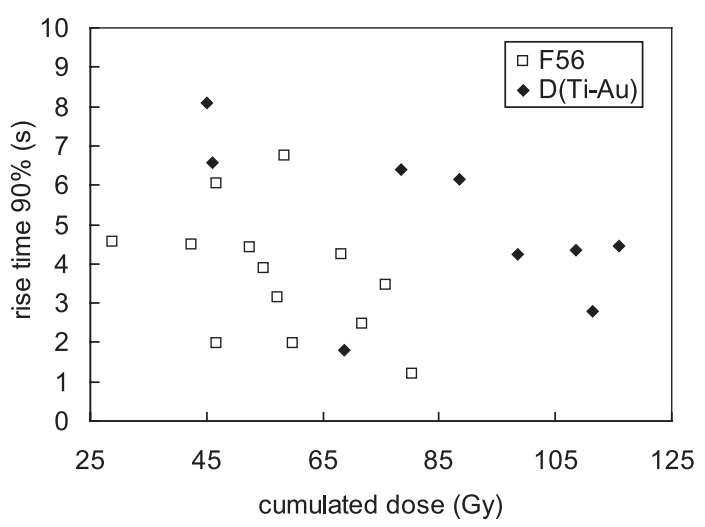

Figure 4. Rise time, $90 \%$ of the equilibrium current, vs. cumulated dose. Signals were recorded after stability of the response was achieved for both detectors. Rise times are determined at different dose rates. For F56, the data obtained in two runs of measurements performed in two successive days are reported. Uncertainty $(0.1 \mathrm{~s})$ is within the symbol size.

the various irradiation runs and also to the delay between successive irradiations. A dedicated experiment to show the effect of these parameters on the rise time is underway.

\section{CONCLUSIONS}

In this work some dosimetry features of two CVD diamond detectors in a $25 \mathrm{MV} \mathrm{X}$-photons from a linear accelerator are reported. The comparison was carried out between a local sample grown at the University of Florence, before and after neutron irradiation, and a commercial sample produced by
De Beers. The results show that the quality of a CVD diamond detector, concerning dosimetric characteristics, is significantly improved through irradiation with a high fluence of fast neutrons, probably through a mechanism of compensation of the shallow defects, active at room temperature, via the deep traps created by neutron irradiation. Indeed, the performances of the CVD home-made diamond, after neutron exposure, matched with those of the commercial sample. In particular, the two detectors did not show any significant difference in terms of stability, dynamic of response and dose rate dependence.

\section{REFERENCES}

1. Whitehead, A. J., Airey, R., Buttar, C. M., Conway, J., Hill, G., Ramkumar, S., Scarsbrook, G. A., Sussmann, R. S. and Walker, S. CVD diamond for medical dosimetry applications. Nucl. Instrum. Methods Phys. Res. A 460, 20-26 (2001).

2. Cirrone, G. A. P., Cuttone, G., Raffaele, L., Sabini, M. G., De Angelis, C., Onori, S., Pacilio, M., Bucciolini, M., Bruzzi, M. and Sciortino, S. Natural and CVD type diamond detectors as dosimeters in hadrontherapy applications. Nucl. Phys. A 125, 179-183 (2003).

3. Marczewska, B., Olko, P., Nesladek, M., Waligorsky, M. P. R. and Karremans, Y. CVD diamonds as thermoluminescent detectors for medical applications. Radiat. Prot. Dosim. 101, 485-488 (2002).

4. Bruzzi, M., Bucciolini, M., Casati, M., DeAngelis, C., Lagomarsino, S., Løvik, I., Onori, S. and Sciortino, S. CVD diamond particle detectors used as on-line dosimeters in clinical radiotherapy. Nucl. Instrum. Methods Phys. Res. A 518, 421-422 (2004).

5. Pini, S., Bruzzi, M., Bucciolini, M., Borchi, E., Lagomarsino, S., Menichelli, D., Miglio, S., Nava, F. and Sciortino, S. High-bandgap semiconductor 


\section{DE ANGELIS ET AL.}

dosimeters for radiotherapy applications. Nucl. Instrum. Methods Phys. Res. A 514, 135-140 (2003).

6. Bruzzi, M., Bucciolini, M., Cirrone, G.A.P., Cuttone, G., Mazzocchi, S., Pirollo, S. and Sciortino, S. Characterization of CVD diamond dosimeters in on-line configuration. Nucl. Instrum. Methods Phys. Res. A 454, 142-146 (2000).

7. Bruzzi, M., Menichelli, D., Pini, S., Bucciolini, M., Molnar, J. and Fenyvesi, A. Improvement of the dosimetric properties of chemical-vapor-deposited diamond films by neutron irradiation. Appl. Phys. Lett. 81, 298-300 (2002).

8. Bacci, T., Borchi, E., Bruzzi, M., Santoro, M. and Sciortino, S. Correlation between material properties and leakage currents in CVD diamond films deposited by DC plasma glow discharge. Mater. Sci. Eng. B 47, 54-63 (1997).

9. Fowler, J. F. Solid state electrica conductivity dosimeters. In Radiation Dosimetry. Attix, F. H. and Roesch, W. C. Eds (NY: Accademy) (1966).

10. Bruzzi, M., Miglio, S., Pirollo, S. and Sciortino, S. Electrical properties and defect analysis of neutron irradiated undoped CVD diamond films. Diam. Rel. Mat. 10, 601-605 (2001).

11. De Angelis, C., Onori, S., Pacilio, M., Cirrone, G. A. P., Cuttone, G., Raffaele, L., Bucciolini, M. and Mazzocchi, S. An investigation of the operating characteristics of two PTW diamond detectors in photon and electron beams. Med. Phys. 29, 248-254 (2002). 